\title{
Pattern Graphs: Combining Multivariate Time Series and Labelled Interval Sequences for Classification
}

\author{
Sebastian Peter, Frank Höppner and Michael R. Berthold
}

\begin{abstract}
Classifying multivariate time series is often dealt with by transforming the numeric series into labelled intervals, because many pattern representations exist to deal with labelled intervals. Finding the right preprocessing is not only time consuming but also critical for the success of the learning algorithms. In this paper we show how pattern graphs, a powerful pattern language for temporal classification rules, can be extended in order to handle labelled intervals in combination with the raw time series. We thereby reduce dependence on the quality of the preprocessing and at the same time increase performance. These benefits are demonstrated experimentally on 10 different data sets.
\end{abstract}

\section{Introduction}

In recent years the development of cheaper sensors and bigger storage capacities has led to an increase in the amount of data gathered periodically. Companies are now able to use (mobile and/or wireless) sensor networks more efficiently in many different domains (e.g. health care, climate, traffic, business processes to name a few) to collect data with usually various dimensions. By analysing temporal data, companies try to gather more insight into their processes and are thereby able to draw

S. Peter $(\varangle) \cdot$ M. R. Berthold

Nycomed-Chair for Bioinformatics and Information Mining, University of Konstanz, Box 712, D-78457 Konstanz, Germany

e-mail: sebastian.peter@uni-konstanz.de

M. R. Berthold

e-mail: michael.berthold@uni-konstanz.de

F. Höppner

Department of Computer Science, Ostfalia University of Applied Sciences,

D-38302 Wolfenbüttel, Germany

e-mail: f.hoeppner@ostfalia.de 
conclusions, enabling them for example, to predict the market for the next week or optimise the output by improving the production process.

One important aspect during the analysis step is often finding typical or characteristic situations. To grasp or encompass these situations, various notions of multivariate temporal patterns are described in literature. Example applications for multivariate temporal patterns include the discovery of dependencies in wireless sensor networks [1], the exploration of typical (business) work flows [3] or the classification of electronic health records [2]. Temporal patterns are often applied to labelled interval data, as the resulting patterns are easy to understand for the experts and also allow us to deal with multivariate data. To incorporate numerical time series in the patterns, they are discretized and their behaviour is described by a linguistic term ('low revolutions', 'slowly accelerating') that holds over a given period of time, hence the term 'labelled (temporal) interval'. The effectiveness of such patterns depend strongly on this discretization step. In this paper we extend the powerful concept of pattern graphs (see Fig. 1 as an example) enabling us to deal directly with time series data and overcome the sensitivity of the preprocessing phase.

The paper is outlined as follows: The next section reviews related work and further motivations for our work. We then give an introduction to pattern graphs (Sect.3) and the matching and learning algorithms (Sect.4) [10, 11]. In Sect. 5, we contribute the necessary changes to incorporate numeric time series. Section 6 presents the experimental results, and we conclude the paper in Sect. 7.

\section{Motivation and Related Work}

In this paper we concentrate on multivariate temporal patterns to characterise the evolution of multiple variables over time. These patterns are used in the antecedents of classification rules. The data consists of labelled temporal intervals; the labels may address categorical (e.g. 'gear-shift' in Fig. 1) or numerical features (e.g. 'low revolutions' in Fig. 1). These labelled intervals and their relationships are combined to form temporal patterns, for example by specifying the relationships between all observed intervals like ' $A$ before $B$ ', 'A overlaps $C$ ' and ' $C$ overlaps $B$ ' $[2,5]$. This notation is quite strict and somewhat ambiguous [7], because the qualitative

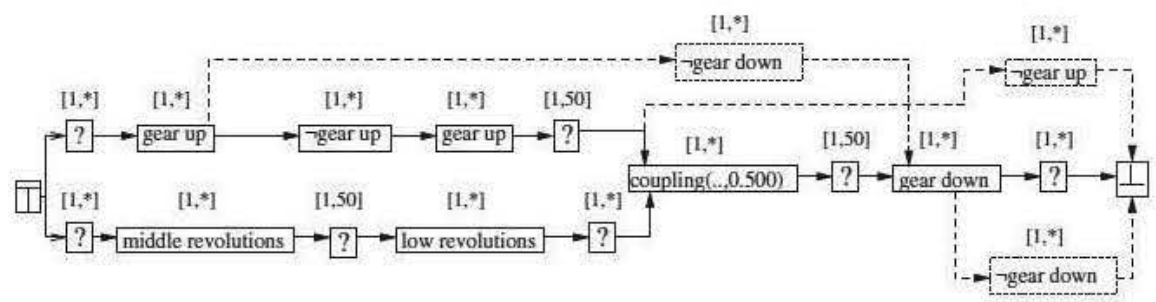

Fig. 1 Example of a pattern graph describing a driving cycle (learned from data, see [10]) 
relationship does not carry quantitative information about the degree of overlap or size of a gap. Other approaches contain such information [3], but consider only those events that do not include durations and thus offer no means to express concurrency as in ' $A$ and $B$ must co-occur for 5-10 time units'. To be more robust against noise, some approaches allow to address parts of the intervals only [4, 9]. The recently proposed pattern graphs [11] satisfy most of the shortcomings and will be used in this paper (and will be introduced below in more detail).

Regardless of the pattern language, when the recorded data is numeric in nature, this leads to the problem of having to convert the numeric data into labelled intervals. This is usually done by applying thresholds, clustering methods or methods dedicated to finding stable intervals (e.g. 'Persist' [8]). This step is time consuming: multiple iterations and manual inspections are needed for a suitable discretization as a bad discretization can render all efforts to retrieve good patterns useless. An example is shown in Fig. 2, where the values of two time series (a) and (b) are discretized using the threshold 5, leading to the same sequence of labelled intervals (with labels [low: $y \leq 5$ ] and [high: $y>5$ ]) in Fig. 2c. In this case the sequences are not distinguishable anymore, which is undesired if both series belong to different classes and we look for a temporal pattern that distinguishes both classes from each other. Furthermore the one perfect discretization may not exist in a situation where in class (a) the threshold needs to be 5 whereas for class (b) 6 and for class (c) the threshold of 7 would be perfect. To overcome this problem, the selection of optimal thresholds may be postponed in the learning algorithm itself instead of leaving it as a preprocessing step.

\section{Pattern Graphs}

This section reviews pattern graphs, which were first introduced in [11]. We consider $m$ (categorical or numeric) attributes with value range $D_{j}$, composed of multivariate observations $\mathbf{x} \in D$ with $D=\left(D_{1} \times \cdots \times D_{m}\right)$.

(a)

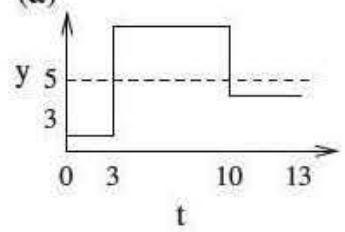

(b)

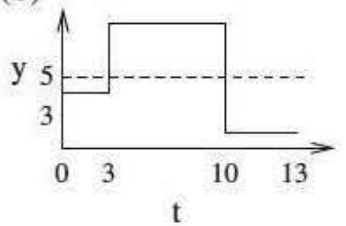

(c)

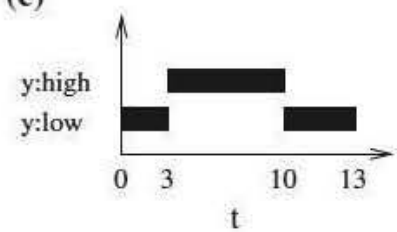

Fig. 2 Two time series: positive class (a) and negativie class (b) discretized to the interval sequence (c) by using the threshold 5 (dotted line), thereby loosing information to distinguish them from each other 
Definition 1 ((sub)sequence). A sequence $S$ consists of an arbitrary number of observations $\left(\mathbf{x}_{1}, \ldots, \mathbf{x}_{n}\right) \in \mathscr{S}$ with $\mathscr{S}=\bigcup_{i=1}^{\infty} D^{i}$. Let $|S|=n$ denote the length of the sequence $S$. A subsequence from index $a$ to $b$ of $S$ is denoted by $\left.S\right|_{[a, b]}$.

To describe those parts of the series that are relevant for the classification task, we apply (local) constraints to subsequences:

Definition 2 (set of constraints for (sub)sequences). Let $\mathscr{C}=\{C \mid C: \mathscr{S} \rightarrow$ $\mathbb{B}\}$ denote the set of all (possible) constraints on (sub)sequences. We distinguish between value-constraints, restricting the acceptable values of the (sub)sequence, and temporal constraints, which limit their duration. For a sequence $S=\left(\mathbf{s}_{1}, \ldots, \mathbf{s}_{k}\right)$, examples of value-constraints are:

- $C(S)=$ true ("don't care": is always satisfied)

- $C(S)=$ true $\Leftrightarrow \forall i: 1 \leq i \leq k: s_{i, j} \in C_{j}$ with $C_{j} \subseteq D_{j}$ for all $1 \leq j \leq m$. This constraint limits the range of accepted values for the sequence.

In this paper we consider only one type of temporal constraint:

- Given $t \in T, T=\{(a, b) \mid 1 \leq a \leq b\} \subseteq \mathbb{N}^{2}$, a temporal constraint is defined as $C(S)=$ true $\Leftrightarrow a \leq|S| \leq b$. Therefore a temporal constraint is represented by an interval $[a, b]$ and restricts the duration of the (sub)sequence $S$ to lie within these bounds. Here $a$ is considered the minimal and $b$ the maximal temporal constraint.

Up to now, pattern graphs have only been used for interval sequences, that is, a condition (described by the interval label) either holds or not $\left(D_{j}=\{0,1\}\right)$. We thus have three different value-constraints: $C_{j} \subseteq\{0\}$ (absent), $C_{j} \subseteq\{1\}$ (present) and $C_{j} \subseteq\{0,1\}$ (don't care). A pattern graph defines a partial order of constraints:

Definition 3 (pattern graph). A tuple $M=\left(V, E, \mathscr{C}_{\text {val }}, \mathscr{C}_{\text {temp }}\right)$ is a pattern graph, iff $(V, E)$ is an acyclic directed graph with exactly one source $(\top)$, one sink $(\perp)$, a finite node set $V \subseteq \mathbb{N} \cup\{\top, \perp\}$ and an edge set $E \subseteq(V \times V)$, for which the following properties hold with $V^{\prime}=V \backslash\{\top, \perp\}$ :

- $\forall\left(v_{1}, v_{2}\right) \in E: v_{2} \neq \top$

- $\forall\left(v_{1}, v_{2}\right) \in E: v_{1} \neq \perp$

(no incoming edge to $\top$ )

- $\forall v \in V^{\prime}:(\exists w \in V:(v, w) \in E) \wedge(\exists w \in V:(w, v) \in E)$

(all nodes $v \in V^{\prime}$ have at least one incoming and outgoing edge)

Finally, $\mathscr{C}_{\text {val }}: V^{\prime} \rightarrow \mathscr{C}$ is a function, mapping each node $v \in V^{\prime}$ to a value-constraint $C \in \mathscr{C}$, whereas $\mathscr{C}_{\text {temp }}: V^{\prime} \rightarrow \mathscr{C}$ maps each node $v \in V^{\prime}$ to a temporal constraint $C \in \mathscr{C}$. By $\mathscr{C}_{\text {val }}^{v}$ we abbreviate $\mathscr{C}_{\text {val }}(v)$, i.e., the value constraint assigned to $v$. We define $\mathscr{C}_{\text {temp }}^{v}:=\mathscr{C}_{\text {temp }}(v)$ analogously.

Definition 4 (mapping). A mapping $B$ for a sequence $S$ and a pattern graph $M=\left(V, E, \mathscr{C}_{\text {val }}, \mathscr{C}_{\text {temp }}\right)$ assigns each node $v \in V \backslash\{\top, \perp\}$ a continuous subsequence $\left.S\right|_{[a, b]}$. T is assigned the fictitious subsequence $\left.S\right|_{[0,0]}$ and $\perp$ the subsequence $\left.S\right|_{[|S|+1,|S|+1] .} B(v)=[a, b]$ denotes the start and end index of the subsequence of $\mathrm{S}$ assigned to node $v$. 
(a)

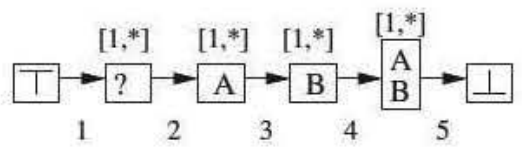

(b)

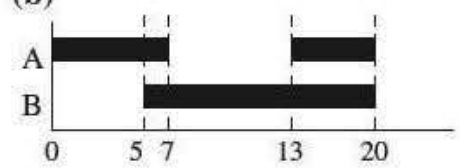

Fig. 3 Example for multiple mapping candidates on a given graph (a) and sequence (b)

Definition 5 (match, valid mapping). A valid mapping $B$ for pattern graph $M=$ $\left(V, E, \mathscr{C}_{\text {val }}, \mathscr{C}_{\text {temp }}\right)$ and a sequence $S$ of length $n$ is a mapping with the following additional properties: with $V^{\prime}=V \backslash\{T, \perp\}$

$$
\begin{aligned}
& \forall\left(v_{1}, v_{2}\right) \in E: B\left(v_{1}\right)=[a, b] \wedge B\left(v_{2}\right)=[c, d] \Rightarrow b+1=c \quad \text { (no gaps) } \\
& \forall i: 1 \leq i \leq n: \exists v \in V^{\prime}: i \in B(v) \text { (each index is assigned at least once) } \\
& \forall v \in V^{\prime}: \mathscr{C}_{v a l}^{v}\left(\left.S\right|_{B(v)}\right)=\text { true (value-constraint holds) } \\
& \forall v \in V^{\prime}: \mathscr{C}_{\text {temp }}^{v}\left(\left.S\right|_{B(v)}\right)=\text { true (temporal constraint holds) }
\end{aligned}
$$

Having defined the pattern graph in detail we will now give an example to illustrate the semantics of the pattern graph. Figure 3a shows an example of a pattern graph with one path, which is read as follows: The temporal constraint of a node is depicted above the node. A star represents an unlimited duration. The value-constraint of a node is shown inside the node. We have two kinds of value-constraints for attribute $A$ : $A$ means that the attribute is active $\left(D_{A}=\{1\}\right)$ and $\neg A$ requires its absence $\left(D_{A}=\{0\}\right)$. A node labelled '?' (don't care) is unconstrained. Please note that if the node states $A$ the behaviour of the other attributes is unconstrained.

Figure $3 b$ shows a sequence where the vertical axis reveals two attributes $A$ and $B$, which hold over certain periods of time (black bars, time on horizontal axis). We now discuss whether these sequence match the pattern graph in Fig. 3a. As this is a simple graph, it contains only one path from source to sink. For this graph the sequence has to be divided into four contiguous parts, so that the first part satisfies the 'don't care' constraint; during the second part the property $A$ has to hold; the property $B$ must hold in the third part and both $A$ and $B$ have to hold during the last part. All of these four parts require a duration of at least one time unit (but have no upper bound on the duration) except the $A$ node with a minimum duration of 3 . The sequence shown in Fig. 3a can be mapped to the graph, because we can clearly see that $A$ is active until $B$ begins and is active until the end and during the last part $A$ becomes active again through to the end of the sequence. Actually the pattern graph has more than one valid mapping (discussed later in Sect.4). A more complex and expressive pattern graph is found in Fig. 1, which describes a driving cycle derived from real data [10]. 


\section{Matching and Learning Pattern Graphs}

Matching a pattern graph to a sequence is essentially a combinatorial problem, an efficient matching algorithm can be found in [11]. Often multiple matches are possible and for each edge $e=(u, v) \in E$ the algorithm provides a set of valid edge positions $p(e)$, i.e., a set of positions $t$ that satisfy all value-constraints of node $u$ for $t^{\prime}<t$ and all value-constraints of node $v$ for $t^{\prime} \geq t$. For the graph in Fig. 3a and the sequence in Fig. 3b we have a set of valid locations $p(e)=\{0\}$ for the edge $e$ from node $\top$ to $\perp$, but for the edge $e^{\prime}$ from $B$ to $A B$ we have $p\left(e^{\prime}\right)=\{13,14, \ldots, 19\}$. These edge positions are organized in so called mapping candidates, which map each edge of the graph to one contiguous interval of valid edge positions. So a mapping candidate $C$ may be considered as a precursor of a valid mapping $B$ in the following sense (by $(\cdot, v)$ we denote any edge leading to $v)$ :

$$
\forall v \in V: B(v)=[s, e] \Rightarrow s \in C(\cdot, v) \wedge e \in C(v, \cdot)
$$

Multiple mapping candidates exist, from which one or more valid mappings may be derived. Consider the graph in Fig. 3. The '?' node is valid during [0,20], the 'A' node is satisfied during [0,7] and [13,20], 'B' during [5,20] and finally 'AB' during $[5,7]$ and $[13,20]$. Out of these sets of valid positions the matching algorithm derives two mapping candidates $C_{1}$ and $C_{2}$, assigning each edge its admissible positions. Three valid mappings $B_{1}-B_{3}$, obtained from $C_{1}$ and $C_{2}$, are shown below (many more are possible).

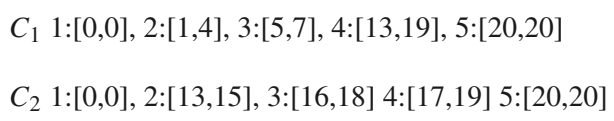

While all edge positions from the mapping candidates ensure that the value constraints hold, the positions must also fulfil the temporal constraint of the node. For instance, if the temporal constraint for the node labelled 'A B' was [5, *], the mapping $B_{2}$ and $B_{3}$ would no longer be valid (because the sequence assigned to this node has only a length of $\leq 4$ ).

A two-phased learning algorithm for pattern graphs has been introduced in [10], where a general pattern, matching all instances of a class, is learned in the first phase. The second phase implements a beam-search, where in each iteration, the $k$-best pattern graphs are refined further by special refinement operators, which add new nodes or edges to the graph, modify temporal constraints or add value constraints to nodes in order to improve some measure of interestingness (we apply the J-measure [12]). 
(a)

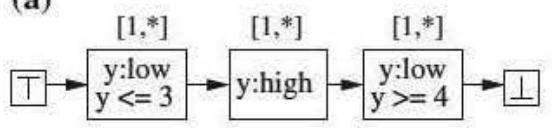

(b)

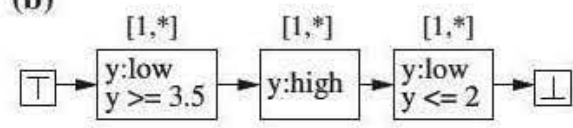

Fig. 4 Two pattern graphs with a new constraint to distinguish the positive between the negative sequences from Fig. 2

\section{Extending Pattern Graphs to Time Series}

In order to enable pattern graphs to deal with a numeric range $D_{j} \subseteq \mathbb{R}$ (cf.Def.1) we introduce new value constraints called series constraints, where $C_{j}=\{x \mid x \leq$ $\sigma\} \subseteq D_{j}$ or $C_{j}=\{x \mid x \geq \sigma\} \subseteq D_{j}$ for some threshold $\sigma$ (cf.Def. 2). With these additional constraints we enable pattern graphs to overcome the obstacle of finding the best discretization to convert time series to labelled intervals, as every node may now use its own threshold $\sigma$ instead of relying on the predefined intervals alone. This enables us to use different thresholds for different classes and it also allows us to use different series constraints for the same series within the same class (local constraints in different nodes). For example we can create the pattern graphs shown in Fig. 4a and $b$ which are able to separate the sequences shown in Fig. 2a and b nevertheless they have the same interval representation as shown in Fig. 2.

To learn such constraints automatically from data, we have to extend the beam search operators. While it is quite easy to check whether a given assignment of subsequences to graph nodes represents a valid mapping, it is much more complicated to derive new conditions that are in some way 'optimal' for the set of all possible mappings. This is due to the fact that a graph node with constraint ' $y$ :low' may match an interval $[s, e]$ with this label in many different ways: any sub-interval $\left[s^{\prime}, e^{\prime}\right] \subseteq[s, e]$ satisfies the node constraints; such a constraint still leaves many possibilities for valid mappings. In order to refine (or introduce new) node constraints, we have to consider all of these potential mappings at the same time, in order to calculate what would be the best additional constraint to distinguish good from bad cases. Enumeration of all possible mappings is not feasible because of their large number. Thus we operate directly on the mapping candidates of the matching algorithm.

Without loss of generality we will consider only the $x \leq \sigma$ constraint in the following. The new operator is instantiated for each individual graph node $v \in V$. As with all the other operators, it receives all mapping candidates (that already reflect the value constraints of that node), the temporal constraint and the data sequence. The objective is to derive a threshold $\sigma$ on one (numeric) variable $x$ of the sequence, such that the additional node constraint $x \leq \sigma$ improves the discriminative power of the pattern. Expressed more formally: if $P$ is a pattern graph, let $m_{P}(s)=1$ denote that $P$ has a valid mapping to $s \in S$ (0, else). Let $G^{\prime}$ denote the resulting pattern if $P$ is extended by the constraint $x \leq \sigma$ in node $v$. Then a confusion matrix from $m_{G^{\prime}}(S) \in\{0,1\}$ and a class $k / \neg k$ is created for $G^{\prime}$ to evaluate its utility. 
The naive approach to find the best refinement is to extend $P$ with every possible series constraint for $x$ and then match all of the resulting graphs to all sequences. It is sufficient to examine only those thresholds $\sigma$ that change the matching result of some $s \in S$, that is, for $\sigma$ we obtain $m_{G^{\prime}}(s)=1$ but for $\sigma-\varepsilon$ we have $m_{G^{\prime}}(s)=0$, because it is only at these thresholds that the confusion matrices of the rule change. Thus, we need to determine only as many confusion matrices as we have sequences. How do we find the threshold $\sigma$ for a given sequence $s$ ? If a subsequence, mapped to node $v$, shall satisfy the constraint, we have to choose $\sigma$ as the maximum of all $x$. However we do not know this subsequence in advance, but have to consider all possible subsequences that may be obtained from the mapping candidates. To let all subsequences satisfy the constraint, we have to pick the smallest of all maximum values of all possible subsequences. If this value were reduced only slightly $(-\epsilon)$, there would be at least one subsequence for node $v$ that would not match anymore with the result that no valid mapping exists anymore. Lemma 1 shows that it is sufficient to inspect only the shortest possible subsequences rather than all possible subsequences.

Lemma 1. By max $S$ we denote the maximum of the $x$-values in a (sub) sequence $S$. Let $\mathscr{Q}$ be the set of all subsequences (that may occur in a valid mapping to node $v)$ and $\mathscr{Q}^{\prime}$ the set of shortest subsequences. ${ }^{1}$ Then $\min \max _{S \in \mathscr{Q}} S=\min \max _{S \in \mathscr{Q}^{\prime}} S$ holds.

Proof. Let $S \in \mathscr{Q}$. Without loss of generality let us assume that $S \notin \mathscr{Q}^{\prime}$. Thus, $S$ is not among the shortest subsequences and therefore we find a $T \in \mathscr{Q}^{\prime} \subseteq \mathscr{Q}$ such that $T$ is a subsequence of $S$. All values of $T$ are contained in $S$, but $S$ contains additional entries, therefore we have $s:=\max S \geq \max T=: t$. Thus, we know that $\min \max _{S \in \mathscr{Q}^{\prime}} S \leq t \leq s$. This means, that $s=\max S$ can be ignored safely in the calculation of $\min \max _{S \in \mathscr{Q}} S$.

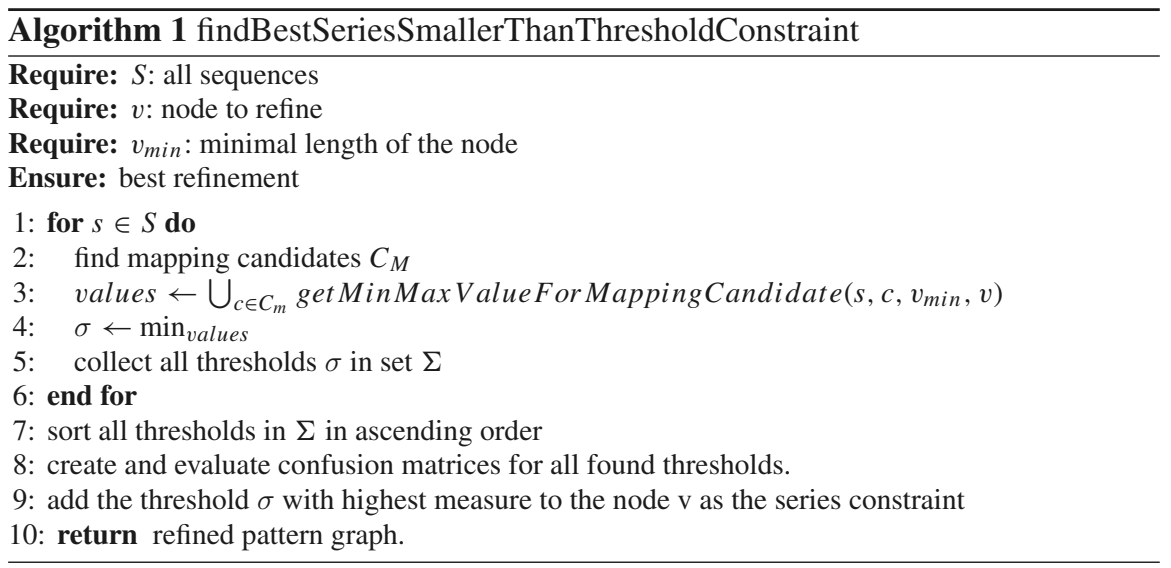

\footnotetext{
${ }^{1}$ shortest in the following sense: $\forall s^{\prime} \in Q^{\prime}: \neg \exists s \in Q: s \subset s^{\prime}$.
} 


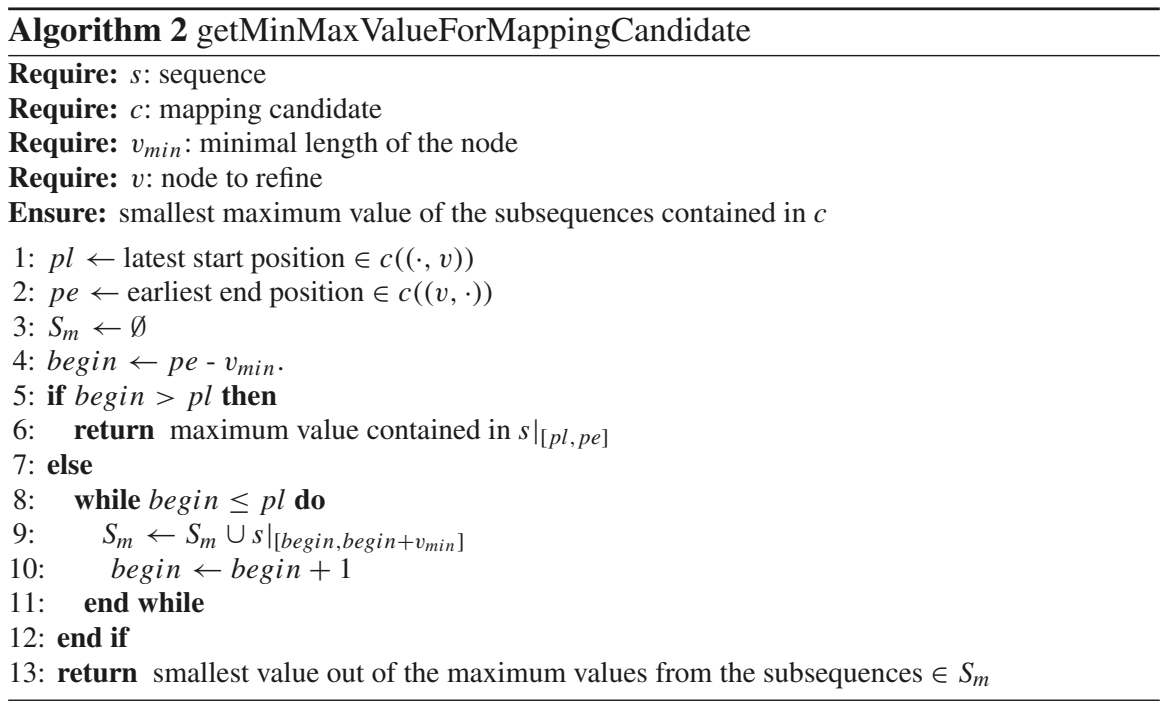

The outline of the refinement operator to find the best $x \leq \sigma$ is shown in Algorithm 1. In the lines 1-6 the algorithm computes the threshold as defined by the Lemma 1. It utilises Algorithm 2 to find the maximum value of all shortest subsequences for a given mapping candidate. $v_{\min }$ denotes either the minimal temporal constraint of node $v$, or is a greater value if the graph structure requires longer sequences in order to satisfy the temporal constraints of other nodes (for example due to parallel paths).

We find the best refinement by evaluating all possible confusion matrices and picking the one with the highest interestingness measure. In order to avoid overfitting, the series constraint with the best measure will be relaxed similarly to the binary split operator in decision tree learning: We search for the next greater value and use the mean of both. This doesn't change the prediction of the new pattern on the training set, but is less restrictive for new instances. The refinement is completed in line 9 by adding the series constraint with the computed value to the node.

From Algorithm 2 we can see that the shortest subsequences for a single mapping candidate are always subsequences with the same length, shifted by one time unit. This allows us to use a priority queue, in order to extract the constraint value efficiently.

Overfitting. An important step to avoid overfitting is to prevent nodes with the minimum temporal constraints 1 to be refined with a series constraint. This would allow the pattern graph to focus on one single time point and would thus stimulate overfitting. We therefore enforce a minimal length of a node to be refined with the new series constraint. If a node has a minimal duration of 1 during refinement, the minimal length will be set to this minimal length (lower bound of $v_{\min }$ ). This has the consequence for step 1 that only subsequences with the minimal length, which are mappable to the node have do be analysed. Additionally we have added a likelihood 
ratio [6] test after every refinement and keep only those graphs with statistically significant improvements to avoid overfitting (which is a problem common to all rule learners).

\section{Experimental Evaluation}

The experimental evaluation is divided into two different experimental setups. In the first experiment we show that the series constraints help to overcome the preprocessing problem discussed earlier. Whereas in the second experiment we show that the new approach is able to perform better, even if a good discretization is applied beforehand.

\subsection{Robustness Against Preprocessing Errors}

To show that series constraints could help dealing with sub-optimal preprocessing of the data we took nine data sets from the UCR time series repository. ${ }^{2}$ This repository already supplies training and tests partitions for each data set in a common format. All of these data sets consist of a raw univariate time series which requires some preprocessing: a moving average smoothing was applied to the series and we also extracted an additional slope series. Thereby we artificially converted the data into a multivariate time series (original and slope time series). In the second step this preprocessed time series had to be converted into a labelled interval series by applying 3-quantile discretization. To achieve different discretization the quantile boundaries are selected randomly for each iteration. We are aware of the fact that for the given data sets algorithms exists that perform better, but most of these approaches could not deal with multivariate data. These approaches often utilize 1-nearest neighbor classification $(1 \mathrm{NN})$ with Euclidean distance or dynamic time warping, whereas the pattern graphs rely on simple elements only (like intervals with a value $\geq \sigma$ ) and thus highlight structural differences. These simple elements keep pattern graphs interpretable even in the case of complex multivariate data (see Sect. 6.2). Therefore $1 \mathrm{NN}$-approaches are not the real competitors. To show the improvement of the learned graphs with series constraints and allow future comparison for follow up work we decided to use these data sets. Table 1 displays the results obtained by applying the beam with and without the series constraints for 30 iterations per class and dataset. The parameter for the minimum sequence length was set to 10, but the results obtained by using additional operators with 5,15 and 20 as minimal length led to nearly the same results. The first row names the dataset, the second row displays the class (for which the pattern graphs was learned for). The left side represents the search without

\footnotetext{
${ }^{2}$ Keogh, E., Zhu, Q., Hu, B., Hao. Y., Xi, X., Wei, L. \& Ratanamahatana, C. A. (2011). The UCR Time Series Classification/Clustering Homepage: www.cs.ucr.edu/ eamonn/time_series_data/.
} 
Table 1 Results on the data when the thresholds vary

\begin{tabular}{|c|c|c|c|c|c|c|c|c|c|c|c|c|}
\hline \multirow{2}{*}{$\begin{array}{r}\text { Data set } \\
\text { Class }\end{array}$} & \multicolumn{2}{|c|}{ Gun Point } & \multicolumn{4}{|c|}{ Waver } & \multicolumn{4}{|c|}{ Yoga } & \multicolumn{2}{|c|}{ Coffee } \\
\hline & 1 & 2 & -1 & & 1 & & 1 & 1 & 2 & 2 & 1 & 2 \\
\hline Accuracy & \begin{tabular}{|l|l|}
67,5 & 79 \\
\end{tabular} & \begin{tabular}{|l|l|}
61,3 & 75,1 \\
\end{tabular} & \begin{tabular}{|l|}
16,4 \\
\end{tabular} & \begin{tabular}{l|l}
91 & 5
\end{tabular} & \begin{tabular}{l|l}
58,6 & 7
\end{tabular} & 77,5 & 48,2 & 56,7 & 51,4 & 52,7 & \begin{tabular}{|l|l|}
55,8 & 60,6 \\
\end{tabular} & \begin{tabular}{|l|l|}
59,5 & 71 \\
\end{tabular} \\
\hline Std. dev. & \begin{tabular}{|l|l|}
12,3 & 5,7 \\
\end{tabular} & \begin{tabular}{|l|l|}
14,2 & 4,8 \\
\end{tabular} & 21,3 & \begin{tabular}{l|l}
2,7 & 1 \\
\end{tabular} & 10,6 & 0 & \begin{tabular}{|l|}
4,1 \\
\end{tabular} & 2,7 & 4,5 & 3,2 & \begin{tabular}{|l|l|}
13,1 & 1,1 \\
\end{tabular} & \begin{tabular}{|l|l|}
10,3 & 1,8 \\
\end{tabular} \\
\hline Dat: & Light & ng 2 & & & & & & nth & Cor & atrol & & \\
\hline $\mathrm{Cla}$ & -1 & 1 & 1 & & & 2 & & 3 & & 4 & 5 & 6 \\
\hline curacy & \begin{tabular}{l|l}
53,5 & 54,1 \\
\end{tabular} & \begin{tabular}{|l|l|}
51,6 & 56,1 \\
\end{tabular} & 62,5 & 89,3 & 88 & 90,1 & 93,3 & 87,7 & 92,2 & 93,2 & \begin{tabular}{|l|l|}
91,2 & 92,2 \\
\end{tabular} & \begin{tabular}{|l|l|}
87,4 & 94,1 \\
\end{tabular} \\
\hline Std. dev. & 8,1 & 8,2 & 34 & 2,1 & 19,9 & 5,6 & 3 & 5,6 & 2,9 & 3,6 & \begin{tabular}{|l|l|}
3,1 & 3,3 \\
\end{tabular} & 1,4 \\
\hline
\end{tabular}

\begin{tabular}{|c|c|c|c|c|c|c|c|c|c|}
\hline \multirow{2}{*}{$\begin{array}{r}\text { Data set } \\
\text { Class }\end{array}$} & \multicolumn{6}{|c|}{ Two Patterns } & \multicolumn{3}{|c|}{ CBF } \\
\hline & 1 & 2 & & 3 & 3 & 4 & 1 & 2 & 3 \\
\hline Accuracy & \begin{tabular}{|l|l|l|}
34,8 & 96,8 \\
\end{tabular} & 27,79 & 91,1 & 27,4 & 93,2 & \begin{tabular}{|l|l|}
29,8 & 97,5 \\
\end{tabular} & \begin{tabular}{|l|l|l|}
76,3 & 95,9 \\
\end{tabular} & \begin{tabular}{|l|l|}
76,1 & 95,8 \\
\end{tabular} & \begin{tabular}{l|l|}
67 & 77,2 \\
\end{tabular} \\
\hline Std. dev. & \begin{tabular}{|l|l|}
20,6 & 0 \\
\end{tabular} & 13,5 & 2,9 & 13,6 & 1,7 & \begin{tabular}{|l|l|}
18,1 & 0,4 \\
\end{tabular} & \begin{tabular}{|l|l|}
13,5 & 0 \\
\end{tabular} & \begin{tabular}{|l|l|}
11,4 & 0,2 \\
\end{tabular} & \begin{tabular}{|l|l|}
15,1 & 8,5 \\
\end{tabular} \\
\hline
\end{tabular}

\begin{tabular}{|c|c|c|c|c|c|c|c|}
\hline \multirow{2}{*}{$\begin{array}{r}\text { Data set } \\
\text { Class }\end{array}$} & \multicolumn{7}{|c|}{ Fish } \\
\hline & 1 & 2 & 3 & 4 & 5 & 6 & 7 \\
\hline Accuracy & \begin{tabular}{|l|l|l|}
33,9 & 85,7 \\
\end{tabular} & \begin{tabular}{|l|l|}
37,1 & 89,7 \\
\end{tabular} & \begin{tabular}{|l|l|}
42,5 & 91,8 \\
\end{tabular} & \begin{tabular}{|l|l|}
33,7 & 24,9 \\
\end{tabular} & \begin{tabular}{|l|l|}
71,4 & 91,4
\end{tabular} & \begin{tabular}{|l|l|}
28,2 & 86,3 \\
\end{tabular} & \begin{tabular}{|l|l|}
64,7 & 90,4 \\
\end{tabular} \\
\hline Std. dev. & \begin{tabular}{|l|l|}
31,1 & 1,1 \\
\end{tabular} & \begin{tabular}{|l|l|}
34,8 & 0,1 \\
\end{tabular} & \begin{tabular}{|l|l|}
36,1 & 2,1 \\
\end{tabular} & \begin{tabular}{|l|l|}
28,6 & 22,1 \\
\end{tabular} & \begin{tabular}{|l|l|}
34,4 & 3,3 \\
\end{tabular} & \begin{tabular}{|l|l|}
26,3 & 1,2 \\
\end{tabular} & \begin{tabular}{|l|l|}
30,2 & 1,2 \\
\end{tabular} \\
\hline
\end{tabular}

series constraint the right side represents the search with series constraints. The third row indicates accuracy and the fourth contains standard deviation.

For most of the classes the learned pattern graphs with the series constraints are able to perform significantly better in terms of accuracy. We can also see that in most cases standard deviation has decreased, showing that the suboptimal discretization has been compensated. For four classes only small improvements in terms of accuracy and standard deviation occurred. For two classes the performance with series constraints deteriorates: for the class \# 3 from the Synthetic Control data set, standard deviation increases while accuracy drops. In these cases the series constraint led the beam search into a local maximum (the interestingness measure is also lower on the training set). This leaves room for further improvements of this approach, because without the limitations of the beam we should obtain at least the same performance as before.

\subsection{Improved Accuracy on Data with Good Discretization}

We obtained a data set from a German company producing, amongst others, power tools. This data set consists of 8 different classes, where each class describes a different screwing process: screwing in and out using different gears of the power tool and different screws. Each of these 564 instances are described by five time series, e.g. voltage/current at the battery or engine etc. In a first step we manually discretized each of this series to labelled intervals in an interactive manner until we 
Table 2 Results on the power tool data set

\begin{tabular}{|c|c|c|c|c|c|c|c|c|c|c|c|c|c|c|c|c|}
\hline \multicolumn{17}{|c|}{ Power tools } \\
\hline Class & \multicolumn{2}{|c|}{1} & \multicolumn{2}{|c|}{2} & \multicolumn{2}{|c|}{3} & \multicolumn{2}{|c|}{4} & \multicolumn{2}{|c|}{5} & \multicolumn{2}{|c|}{6} & \multicolumn{2}{|r|}{7} & \multicolumn{2}{|c|}{8} \\
\hline Approach & a & b & a & b & a & b & a & b & a & b & a & b & a & b & a & b \\
\hline Accuracy & 98.7 & 98.9 & 98.1 & 99.5 & 98.8 & 98.9 & 98.7 & 98.2 & 97.4 & 99.1 & 96.8 & 97.3 & 98.4 & 98.4 & 95.9 & 99.0 \\
\hline Std. Dev & 0.7 & 0.9 & 0.9 & 0.6 & 0.9 & 0.9 & 0.9 & 1.3 & 1.4 & 1.1 & 1.4 & 1.4 & 1.1 & 1.6 & 2.3 & 2.7 \\
\hline Avg Imp. & \multicolumn{2}{|c|}{0.3} & \multicolumn{2}{|c|}{1.4} & \multicolumn{2}{|c|}{0.01} & \multicolumn{2}{|c|}{-0.5} & \multicolumn{2}{|c|}{1.7} & \multicolumn{2}{|c|}{0.6} & \multicolumn{2}{|r|}{0} & \multicolumn{2}{|c|}{3.1} \\
\hline
\end{tabular}

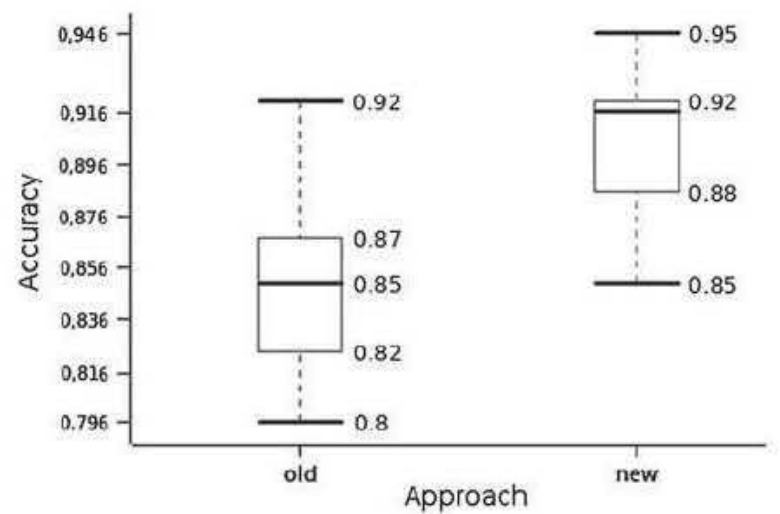

Fig. 5 Box plots showing the results of the complete classifier on the power tool data set

were satisfied with the results. Therefore we may safely assume that discretization is good and it would be hard to achieve better discretization. We applied the beam search (minimal length: 10) to this data set 30 times with and without the series constraint. In each iteration we randomly partitioned the data set into $80 \%$ training and $20 \%$ test. As a result of the good discretization we assume that the accuracy results would be nearly the same, but may be improved by applying different thresholds to one class or in between classes.

Table 2 shows the result. If we sum up the average improvements for all the individual classes, the new graphs performed $6.6 \%$ better. Most of the pattern graphs learned with series constraints perform better (up to $3 \%$ ) and are only slightly worse for one class \#4 $(-0.5 \%)$. However improvements 'per-class' are not significant. So far, each rule has predicted just one class. Next we combine the individual rules to a single, multi-class classifier: we only classify an instance if and only if one pattern graph has a valid mapping on the instance. In case none or more than one pattern graph matches we predict "unknown". The box plot in Fig. 5 and Table 3 shows the result of this classifier, where we use the same pattern graphs as in Table 2, thus the results origin from the same 30 runs with random training and test sets.

We can see that by using series constraints the overall accuracy has improved by an average of $5.8 \%$. It is also interesting to note that in all 30 iterations, the lowest accuracy of the new approach is at least as good as the average result without the series 
Table 3 Mean accuracy and standard deviation of the complete classifier for the powertool dataset

\begin{tabular}{lll}
\hline Approach & Old & New \\
\hline Accuracy & 84.7 & 90.5 \\
Std. Dev & 3.2 & 2.8 \\
\hline
\end{tabular}

constraints. Additionally the mean accuracy using the new approach is nearly equal to the best result obtained without the series constraints $(-1.5 \%)$. By inspecting the learned pattern graphs, we observed that one or two additional series constraints were derived per class. Depending on the class, the thresholds were slightly different, which explains the improved accuracy as the number of false positives was able to be reduced, without increasing the number of false negatives.

\section{Conclusion}

In this paper we have shown, that the results of pattern learning algorithms for labelled sequences rely heavily on discretization of the source time series. The quality of the learned patterns varies considerably when the discretization changes. In order to overcome the problem of finding good discretization, which is time consuming and not always possible, we introduced an algorithm capable of mining labelled intervals together with the corresponding time series. The first experiment has shown that, in comparison to the approach without the series constraint, the quality of the patterns is higher resulting in and allowing for a more robust approach compared to a priori discretization. Furthermore we have shown that even in situations, where discretization already performs well, the quality of the patterns may be increased, because different levels of discretization for different classes and even different thresholds within one class may be utilized.

For future work the synergies of labelled intervals and numeric time series may be improved further as, so far, we have only used simple constraints $(\leq, \geq)$. But it is possible to use more sophisticated constraints on mean values or standard deviation. This kind of constraint may provide further insight into the patterns.

Acknowledgments We would like to thank Stefan Mock from the Robert Bosch GmbH for kindly providing the data.

\section{References}

1. Basile, T.M.A., Mauro, N.D., Ferilli, S., Esposito, F.: Relational temporal data mining for wireless sensor networks (2009).

2. Batal, I., Valizadegan, H., Cooper, G.F., Hauskrecht, M.: A pattern mining approach for classifying multivariate temporal data. In: Bioinformatics and Biomedicine (BIBM), 2011 IEEE International Conference on, pp. 358-365. IEEE (2011). 
3. Berlingerio, M., Pinelli, F., Nanni, M., Giannotti, F.: Temporal mining for interactive workflow data analysis. In: Proceedings of the 15th ACM SIGKDD international conference on Knowledge discovery and data mining, KDD '09, pp. 109-118. ACM, New York, NY, USA (2009).

4. Chen, Y.C., Jiang, J.C., Peng, W.C., Lee, S.Y.: An efficient algorithm for mining time intervalbased patterns in large database. In: Proc. Int. Conf. Inf. Knowl. Mngmt., pp. 49-58. ACM (2010).

5. Höppner, F.: Discovery of temporal patterns - learning rules about the qualitative behaviour of time series. 2168, pp. 192-203. Freiburg, Germany (2001).

6. Kalbfleisch, J.G.: Probability and statistical inference: probability, vol. 2. Springer-Verlag (1985).

7. Mörchen, F.: Unsupervised pattern mining from symbolic temporal data. SIGKDD Explor. Newsl.9(1), 41-55 (2007).

8. Mörchen, F., Ultsch, A.: Optimizing time series discretization for knowledge discovery. In: Proc. Int. Conf. Knowl. Disc. and Data Mining, pp. 660-665. ACM (2005).

9. Mörchen, F., Ultsch, A.: Efficient mining of understandable patterns from multivariate interval time series. pp. 181-215. Springer (2007).

10. Peter, S., Höppner, F., Berthold, M.R.: Learning pattern graphs for multivariate temporal pattern retrieval. In: Proc Int Symp Intel. Data, Analysis (2012)

11. Peter, S., Höppner, F., Berthold, M.R.: Pattern graphs: A knowledge-based tool for multivariate temporal pattern retrieval. In: 6th IEEE International Conference on Intelligent Systems (IS'12) (2012).

12. Smyth, P., Goodman, R.M.: An information theoretic approach to rule induction from databases. IEEE Trans. Knowledge Discovery and Engineering 4(4), 301-316 (1992). 